\title{
A SIMPLE PATTERN RECOGNITION-BASED METHOD FOR POWER QUALITY DISTURBANCE DETECTION
}

\author{
Robson Rosserrani de Lima \\ Center of Instrumentation and Signal Processing (NIPS), PPEE, Federal University of Juiz de \\ Fora (UFJF), Engineering College, Campus, ZIP Code 36.036-900, Juiz de Fora, MG, Brazil. \\ Corresponding author: robson.lima@engenharia.ufjf.br

\section{Danton Diego Ferreira} \\ Federal University of Lavras (UFLA), Automation Department, Campus, ZIP Code 37.200-000, Lavras, MG, Brazil.
}

\author{
José Manoel de Seixas \\ Signal Processing Lab., COPPE/PoLi, Federal University of Rio de Janeiro (UFRJ), Campus, ZIP Code 21.941-972, Rio \\ de Janeiro, RJ, Brazil.
}

\section{Leonardo Silveira Paiva}

Federal University of Lavras (UFLA), Automation Department, Campus, ZIP Code 37.200-000, Lavras, MG, Brazil.

\begin{abstract}
Voltage disturbances are the most frequent cause of a large range of disruption in industrial, commercial, and residential power supply systems. These disturbances are often referred to as power quality problems and affect the Power Systems causing substantial losses. To avoid the storage of a large amount of data, the first task in monitoring the power quality is the realtime detection of disturbances, which must be performed by an accurate and low-complexity system. This paper proposes a low-complexity system for power quality disturbance detection. The method makes innovative use of simple features extracted from reduced segments of the monitored voltage waveform. The extract features (the mean value, variance, energy, and the maximum and minimum values of the filtered voltage signals) require low computational effort and allow a considerable dimensional reduction of the signals, leading to simple detection algorithms. The proposed method achieves high detection rates on both simulated and real signals.
\end{abstract}

Index terms: MLP, Naïve-Bayes, Computational Intelligence.

Received: November, 06, 2020 - Accepted: April, 20, 2021

\section{INTRODUCTION}

Power Quality (PQ) has emerged as an important research field in recent years and the main reasons are: (i) the need to define indices and measures to quantify the quality of the energy delivered; (ii) the wide use of power electronic devices, which are the most important cause of poor PQ; (iii) the need to localize the disturbance sources and (iv) a large amount of PQ data recorded that demands automatic detection (Ribeiro et al., 2013). In this context, $\mathrm{PQ}$ monitoring is required and one of the main reasons is to ensure the availability of power to the customers.

In general terms, the PQ monitoring systems comprise data acquisition, data pre-processing, and data visualization phases. The first step is to measure voltages, to detect real-time voltage disturbances at the Point of Common Coupling (Ribeiro et al., 2013).

Various methods have been proposed in the literature for the disturbance detection and the most used techniques are based on wavelet transform (WT) (Caujolle et al., 2010; Lin et al., 2008; Moravej et al., 2011; Yang and Liao, 2001). However, the results obtained with WT can be seriously affected by system noise, and generally, have highcomputational complexity (Yang and Liao, 2001). The method reported by Thakur and Singh (2013) focused on sag detection and magnitude quantification by using a hybrid concept based on the RMS (Root Mean Square) and peak voltage features. Other methods that should be mentioned include the 
S-transform (Ferreira et al., 2015), the Hilbert transform (Chun-Ling et al., 2009), support vector machines (Marques et al., 2011) and principal curves (Ferreira et al., 2013; Ferreira et al., 2015). Each of these techniques has advantages and disadvantages.

To design automatic disturbance detection systems in Power Systems, an important aspect that must be considered is the computational cost. For real-time applications, low computational cost techniques are preferred. With this purpose, sophisticated techniques have recently been proposed (Ferreira et al., 2013; Ferreira et al., 2015; Marques et al., 2011; Radil et al., 2008). The aim of these detection techniques is to provide a real-time and source reliable detection for a variety of disturbances so that disturbance classification and source identification can be both achieved.

\section{MATERIAL AND METHODS}

This paper proposes a low-computational complexity method for PQ disturbance detection. The method makes innovative use of simple features, which have a good capacity to distinguish between disturbance and nondisturbance conditions. These features are extracted from short segments of the filtered voltage waveform and comprise the mean value, variance, energy, and the maximum and minimum values. For the detection task, the Bayesian Classifier and the Multilayer Perceptron (MLP) are comparatively evaluated on both simulated and real signals.

The paper is organized as follows. The next section presents the PQ disturbance detection formulation. The proposed method is described in Section 3. Section 4 presents the results and discussions and conclusions are derived in Section 5.

\section{PQ Disturbance detection}

The discrete version of the monitored voltage signal can be segmented into non-overlapping frames of $\mathrm{N}$ samples, which are expressed as an additive contribution of several types of phenomena (Ribeiro and Pereira, 2007) as formulated in Equation (1). $\mathrm{v}[\mathrm{n}]=\left.\mathrm{v}(\mathrm{t})\right|_{t=n / f_{\mathrm{s}}}:=\mathrm{f}[\mathrm{n}]+\mathrm{h}[\mathrm{n}]+\mathrm{i}[\mathrm{n}]+\mathrm{t}[\mathrm{n}]+\mathrm{r}[\mathrm{n}]$,

where $n=0, \ldots, \mathrm{N}-1$, fs is the sampling frequency, the sequences $f[n], h[n], i[n], t[n]$ and $r[n]$ are the fundamental component, harmonics, interharmonics, transient and background noise, respectively.

Considering the vector $\mathbf{v}=[v[n] \ldots v[n-\mathrm{N}-1]]^{T}$ built from samples of the signal $v[n]$, the detection problem can be formulated as a hypothesis test problem, represented in Equation (2).

$$
\begin{aligned}
& \mathcal{H}_{0}: \mathbf{v}=\mathbf{f}+\mathbf{r} \\
& \mathcal{H}_{1}: \mathbf{v}=\mathbf{f}+\Delta \mathbf{f}+\mathbf{i}+\mathbf{t}+\mathbf{h}+\mathbf{r}
\end{aligned}
$$

where $\mathbf{i}=[i[n] \ldots i[n-\mathrm{N}-1]]^{T}, \mathbf{t}=[t[n] \ldots t[n-\mathbf{N}-1]]$ ${ }^{T}, \mathbf{h}=[h[n] \ldots h[n-\mathrm{N}-1]]^{T}, \mathbf{r}=[r[n] \ldots r[n-\mathrm{N}-1]]$ ${ }^{T}$. The vector represents a sudden variation in the fundamental component.

The hypothesis $H_{0}$ is related to the nominal operation and the hypothesis $H_{1}$ is associated with abnormal conditions. Note that in this stage, the PQ disturbance detection problem is viewed as a classification problem of two classes, disturbance, and no disturbance.

\section{Method description}

This section describes the details of the steps required to design the proposed low-complexity power quality disturbance detection.

\section{Database}

The proposed method was designed using simulated signals and tested using both simulated and real signals.

The simulated events were generated following the IEEE standards (IEEE, 2009), with a sampling frequency equals to 256 samples per cycle of the fundamental component $\left(f_{s}=15.360 \mathrm{~Hz}\right)$ and SNR (signal-to-noise ratio) equals to $30 \mathrm{~dB}$.

For detection purposes, there are two quite different points of view. The first is to determine whether disturbances exist or not within the processed frame, and the action required is not control or protection, but merely the acquisition of the frame and processing (classification and analysis of the disturbances). In this case, the 
frame size is not so important because there is no immediate or real-time action. However, by assuming a protection application, where a disturbance is detected and identified, the immediate action required is to isolate the system or part of it as quickly as possible. In such a case, the use of a reduced processing frame makes the method more appropriate (Ribeiro et al., 2013).

To evaluate the performance of the proposed method for reduced segments of the monitored voltage signal, segments of the voltage signal are used in the range 16-256 samples, the last corresponding to one cycle of the fundamental component. For each segment size, 110 events were generated for each isolated disturbance. The classes of disturbance considered here were voltage sags, voltage swells, short and long-term voltage interruptions, oscillatory transients, harmonics, transient impulsive and notches, accumulating a total of 880 events. Also, 880 signals without disturbance (nominal voltage signals) were generated. For real signals, 110 voltage waveforms with disturbances provided by the working group of the IEEE (P1159.3) were used (IEEE, 2012).

Of the total data set, $70 \%$ were used for training and $30 \%$ for validation. Using this data set, 100 different training and validation subsets were randomly created (holdout cross-validation procedure). They were generated to evaluate and compare the performance of the classifiers. We used MatLab ${ }^{\circledast}$ software to run the tests. The results are the average \pm standard deviation.

\section{Pre-Processing}

The monitored voltage signals are preprocessed by a notch filter (Hirano et al., 1974), tuned to the fundamental frequency $\left(f_{0}=60 \mathrm{~Hz}\right)$. This filter has been widely used in the literature (Barbosa and Ferreira, 2013; Ferreira et al., 2015; Ferreira et al., 2013; Marques et al., 2011; Ribeiro et al., 2007; Ribeiro and Pereira, 2007) for PQ disturbance detection and classification. This is an important pre-processing step since it removes the fundamental component from the monitored voltage signal, which is the redundant information present in all types of disturbances and also when there are none of these disturbances. Thus, the resulting signal after filtering, here referred to as $\mathbf{e}[n]$, carries information acquired in $\mathbf{v}[n]$ from harmonics, interharmonics, transient, and noise.

Due mainly to the low computational cost and greater selectivity in the frequency of interest, we implement the notch filter using a second-order Infinite Impulse Response (IIR) filter structure (Mitra, 2005), whose Z-transform is given by Equation (3).

$H(z)=\frac{1+a_{0} z^{-1}+z^{-2}}{1+\rho_{0} a_{0} z^{-1}+\rho_{0}^{2} z^{-2}}$

where, so that, and $\rho_{0}$ is the notch factor (here, $\rho_{0}=$ 0.97). The magnitude of the frequency response for this filter is shown in Figure 1.

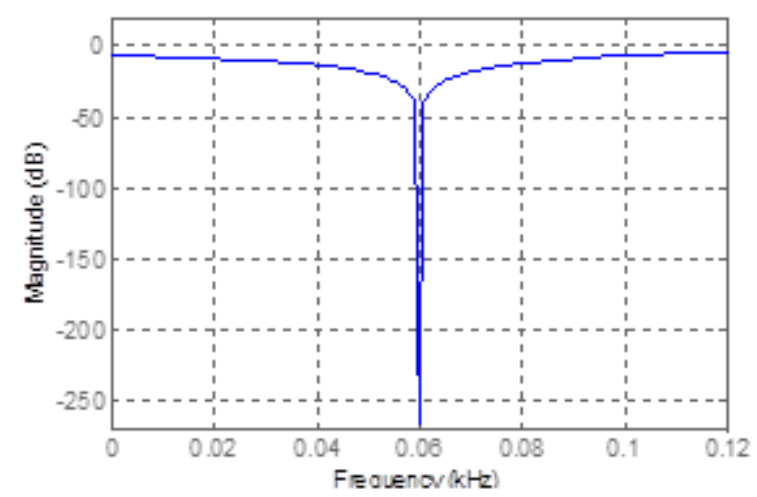

Figure 1: The frequency response of the IIR notch filter used $\left(\rho_{0}=0.97\right)$.

Figure 2 shows pre-processed simulated signals with and without disturbances. Note that when there is no disturbance in the signal, the resulting signal comprises only noise (r) as formulated in (2).

\section{Feature Extraction and Selection}

In order to represent the signals in a reduced dimensional space and maximize the separation border between classes, five features were extracted from $\mathbf{e}[n]$, say: both maximum $\left(\max _{\mathbf{e}[n]}\right)$ and minimum $\left(\min _{\mathrm{e}[n]}\right)$ values, average value $\left(\mu_{\mathbf{e}[n]}\right)$, variance $\left(\sigma_{\mathbf{e}[n]}^{2}\right)$ and energy $\left(E_{\mathbf{e}[n]}\right)$. This last was computed according to (4), where $N$ is the segment size of the monitored signal. This results in a considerable dimension reduction, considering one cycle of the fundamental component. These features were chosen as they are easy to be calculated and we noted that they represent well the disturbances considered. The 
formula used for energy calculation is presented in Equation (4).
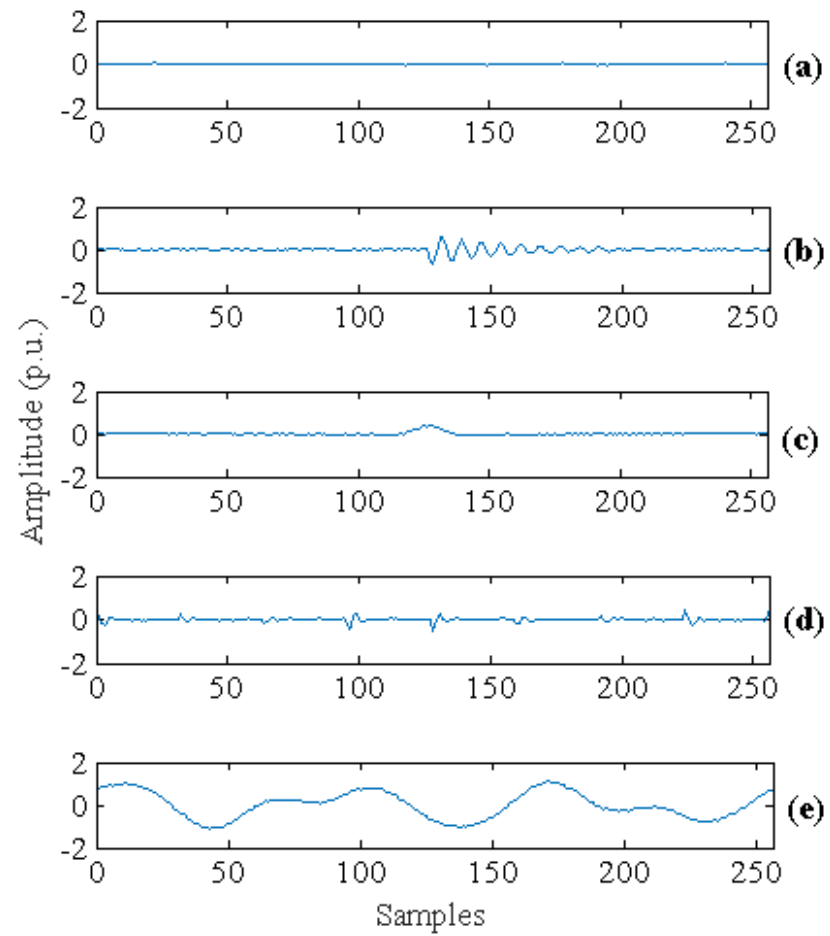

Figure 2: Pre-processed signals (e[ $n]$ ): (a) signal without disturbance, (b) oscillatory transient, (c) voltage sag, (d) notch and (e) harmonics.

$E_{\mathbf{e}[n]}=\sum_{n=1}^{N}|\mathbf{e}[n]|^{2}$

Fisher's discriminant ratio (FDR) has two main and different applications: it can be used as a classifier and as a feature selection tool (Theodoridis and Koutroumbas, 2009) and it is a simple technique for revealing the most relevant features from a multidimensional data. Equation (5) shows the cost function to be minimized in the FDR criterion.

$\mathbf{J}_{\mathbf{c}}=\left(\boldsymbol{\mu}_{1}-\boldsymbol{\mu}_{2}\right)^{2} \odot \frac{1}{\sigma_{1}^{2}+\sigma_{2}^{2}}$,

where $\mathbf{J}_{\mathbf{c}}=\left[J_{1} \ldots J_{\mathrm{L}}\right]^{\mathrm{T}}, L_{l}$ is the total number of features, $\mu_{1}$ and $\mu_{2}, \sigma_{1}^{2}$ and $\sigma_{2}^{2}$ are the vectors of mean and variance of class 1 and class 2 , respectively, where Class 1 refers to the hypothesis $H_{0}$ e Class 2 refers to the hypothesis $H_{1}$, as formulated in (2). The symbol $\odot$ refers to the Hadamard product $\mathbf{r} \odot \mathbf{s}=\left[r_{0} s_{0} \ldots r_{\mathrm{L}-1} s_{\mathrm{L}-1}\right]^{\mathrm{T}}$.

From this, one can understand that the $i$-th feature vector elements, related to higher values of
$\mathbf{J}_{\mathbf{c}^{\prime}}$ represent the features that have a better capacity to distinguish between classes. The potential reduction dimension of $\mathbf{J}_{\mathbf{c}}$ is attractive in systems that require low computational complexity, as proposed here. Furthermore, the use of a reduced number of features can lead to simpler classifiers/ detectors with better performance, since the features responsible for the intersection between classes (or part thereof) can be discarded.

\section{Data Normalization}

Before being presented to classifiers, the feature vectors extracted from the voltage waveform segments were normalized in the range $[-1,1]$ according to the Equation (6).

$\mathbf{x}_{n}=2\left(\frac{\mathbf{x}-\min (\mathbf{x})}{\max (\mathbf{x})-\min (\mathbf{x})}\right)-1$,

where $\mathbf{x}$ is the original feature vector and $\mathbf{x}_{n}$ is its normalized version.

\section{Classifiers}

The extracted features feed the classifiers. Starting from the most relevant features, new features were gradually added to the classifier until including the five features. Two different classifiers are proposed: a linear solution from a Naïve-Bayes classifier (Theodoridis and Koutroumbas, 2009), and a non-linear approach from a Multilayer Perceptron Neural Network (MLP) (Theodoridis and Koutroumbas, 2009).

The MLP network implemented in this work has a single hidden layer and one output neuron. The number of neurons in the hidden layer was varied to determine the best performance. The Resilient Propagation (RPROP) algorithm (Riedmiller and Braum, 1993) for training and the hyperbolic tangent function is the activation function.

\section{RESULTS AND DISCUSSION}

\section{For Simulated Signals}

Figure 3 shows the relevance of each selected feature, according to the FDR criterion from the training set, varying the processing window. 


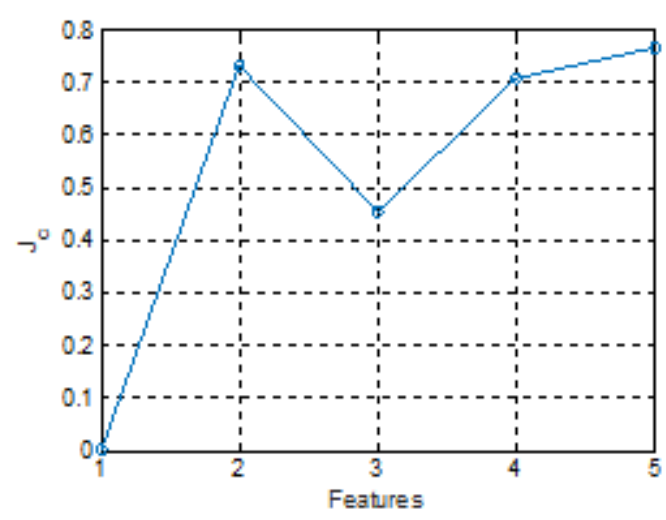

(a)

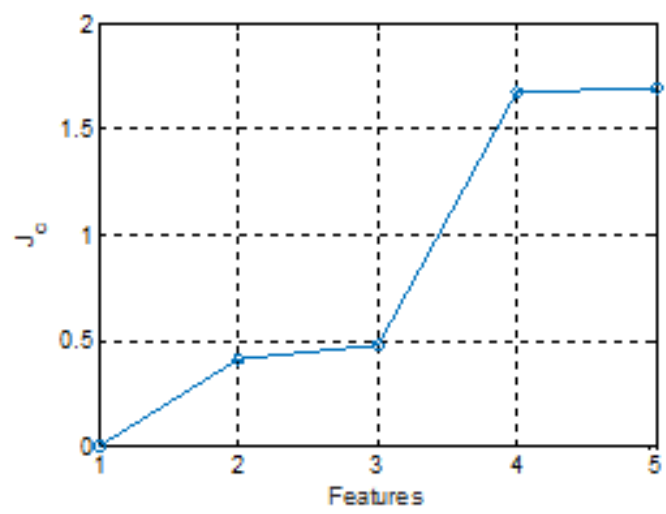

(c)

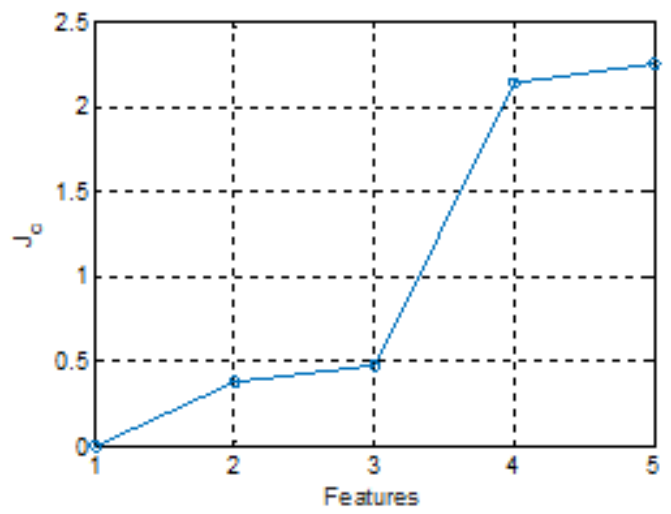

(e)

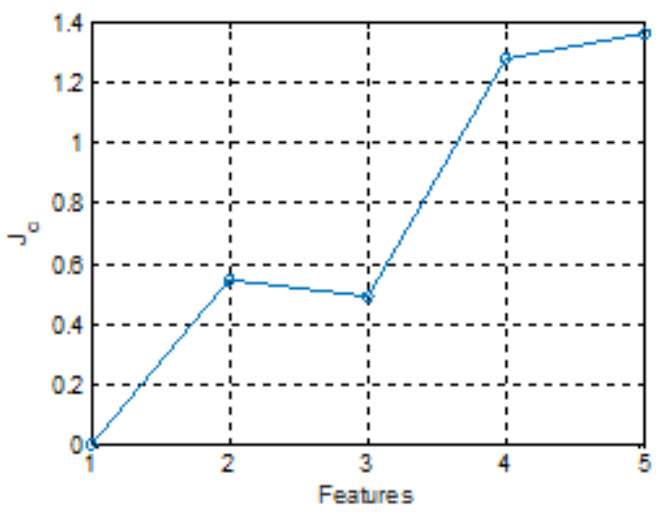

(b)

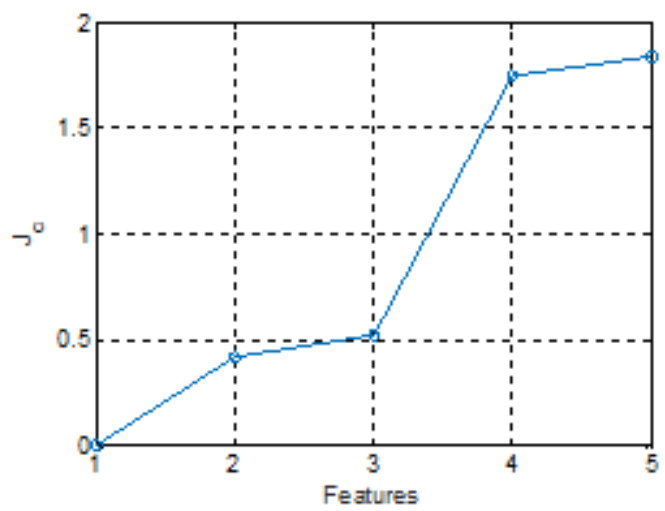

(d)

Figure 3: FDR for signal segments with $N$ samples: (a) $N=16$; (b) $N=32$; (c) $N=64$; (d) $N=128$; (e) $N=256$.

Except when 16 samples are considered, the achieved performance was similar: maximum and minimum values presented greater discrimination capacity concerning the other features. For $N=16$ samples, the variance proved to be as relevant as these two. In all cases, the average value was the feature that contributed the least to the separation between classes.

Tables 1-5 show the performance of the proposed detectors for the validation set for each window length. Class 1 applies to signals with disturbance and Class 2 applies to signals without
PQ disturbances. The results are presented considering feature vectors containing from one to five of the proposed features chosen according to the FDR for each acquisition window.

In general, both Naïve-Bayes and MLP classifiers achieved good detection results. The Naïve-Bayes classifier achieved accuracy rates slightly higher than the MLP mainly for $N<64$ samples and for Class 1 , which comprises the disturbance class.

Figure 4 summarizes the overall efficiency for each classifier in terms of $N$ and the number of extracted features. Regarding the number of 
processed samples, the efficiency increases as $N$ increases. However, it is worth noting that even for $N$ equal to 16 samples (equivalent to
$1 / 16$ of a cycle of the fundamental component), performance above $98.0 \%$ was obtained by the classifiers when four features were used.

Table 1: Detection performance for $N=16$ in $\%$ - simulated data.

\begin{tabular}{ccccc}
\hline \multirow{2}{*}{ Number of Features } & \multicolumn{2}{c}{ Naïve-Bayes } & \multicolumn{2}{c}{ MLP } \\
\cline { 2 - 4 } & Class 1 & Class 2 & Class 1 & Class 2 \\
\hline $1-\max _{\mathrm{e}[n]}$ & $82.07 \pm 4.39$ & $96.45 \pm 2.00$ & $79.64 \pm 11.40$ & $95.41 \pm 9.97$ \\
$2-\sigma_{\mathrm{e}[n]}^{2} \max _{\mathrm{e}[n]}$ & $94.26 \pm 2.56$ & $99.98 \pm 0.11$ & $86.12 \pm 12.08$ & $99.99 \pm 0.11$ \\
$3-\sigma_{\mathrm{e}[n]}^{2}, \min _{\mathrm{e}[n],} \max _{\mathrm{e}[n]}$ & $83.84 \pm 8.53$ & $99.99 \pm 0.04$ & $90.70 \pm 5.01$ & $99.89 \pm 0.30$ \\
$4-\sigma_{\mathrm{e}[n]}^{2}, E_{\mathrm{e}[n]} \min _{\mathrm{e}[n],} \max _{\mathrm{e}[n]}$ & $99.76 \pm 0.86$ & $99.99 \pm 0.04$ & $98.77 \pm 3.05$ & $99.97 \pm 0.14$ \\
$5-\mu_{\mathrm{e}[n]}, \sigma_{\mathrm{e}[n]}^{2}, E_{\mathrm{e}[n]} \min _{\mathrm{e}[n],} \max _{\mathrm{e}[n]}$ & $95.83 \pm 3.47$ & $99.59 \pm 0.76$ & $98.43 \pm 2.02$ & $99.77 \pm 0.78$ \\
\hline
\end{tabular}

Table 2: Detection performance for $N=32$ in $\%$ - simulated data.

\begin{tabular}{ccccc}
\hline \multirow{2}{*}{ Number of Features } & \multicolumn{2}{c}{ Naïve-Bayes } & \multicolumn{2}{c}{ MLP } \\
\cline { 2 - 4 } & Class 1 & Class 2 & Class 1 & Class 2 \\
\hline $1-\max _{\mathrm{e}[n]}$ & $82.74 \pm 9.55$ & $99.78 \pm 1.03$ & $90.60 \pm 6.00$ & $98.59 \pm 3.09$ \\
$2-\min _{\mathrm{e}[n],} \max _{\mathrm{e}[n]}$ & $97.43 \pm 3.48$ & $99.99 \pm 0.06$ & $97.36 \pm 3.62$ & $99.74 \pm 0.95$ \\
$3-\sigma_{\mathrm{e}[n]}^{2}, \min _{\mathrm{e}[n],} \max _{\mathrm{e}[n]}$ & $99.58 \pm 0.68$ & $99.99 \pm 0.04$ & $99.25 \pm 1.40$ & $99.99 \pm 0.06$ \\
$4-\sigma_{\mathrm{e}[n]}^{2}, E_{\mathrm{e}[n]} \min _{\mathrm{e}[n],} \max _{\mathrm{e}[n]}$ & $99.78 \pm 0.58$ & $100.00 \pm 0.00$ & $99.39 \pm 1.33$ & $99.94 \pm 0.28$ \\
$5-\mu_{\mathrm{e}[n]}, \sigma_{\mathrm{e}[n]}^{2}, E_{\mathrm{e}[n]} \min _{\mathrm{e}[n]} \max _{\mathrm{e}[n]}$ & $91.86 \pm 5.32$ & $100.00 \pm 0.00$ & $97.25 \pm 3.75$ & $99.85 \pm 0.42$ \\
\hline
\end{tabular}

Table 3: Detection performance for $N=64$ in $\%$ - simulated data.

\begin{tabular}{ccccc}
\hline \multirow{2}{*}{ Number of Features } & \multicolumn{2}{c}{ Naïve-Bayes } & \multicolumn{2}{c}{ MLP } \\
\cline { 2 - 4 } & Class 1 & Class 2 & Class 1 & Class 2 \\
\hline $1-\max _{\mathrm{e}[n]}$ & $98.63 \pm 2.94$ & $99.21 \pm 1.34$ & $98.55 \pm 3.01$ & $99.76 \pm 0.74$ \\
$2-\min _{\mathrm{e}[n],} \max _{\mathrm{e}[n]}$ & $99.97 \pm 0.15$ & $99.92 \pm 0.29$ & $99.38 \pm 2.22$ & $100.00 \pm 0.00$ \\
$3-E_{\mathrm{e}[n]} \min _{\mathrm{e}[n],} \max _{\mathrm{e}[n]}$ & $100.00 \pm 0.03$ & $99.97 \pm 0.14$ & $99.34 \pm 1.51$ & $99.99 \pm 0.04$ \\
$4-\sigma_{\mathrm{e}[n]}^{2} E_{\mathrm{e}[n]} \min _{\mathrm{e}[n]} \max _{\mathrm{e}[n]}$ & $100.00 \pm 0.00$ & $100.00 \pm 0.00$ & $96.62 \pm 3.23$ & $99.83 \pm 0.99$ \\
$5-\mu_{\mathrm{e}[n]} \sigma_{\mathrm{e}[n]}^{2}, E_{\mathrm{e}[n]} \min _{\mathrm{e}[n]} \max _{\mathrm{e}[n]}$ & $100.00 \pm 0.00$ & $100.00 \pm 0.00$ & $99.59 \pm 0.87$ & $99.99 \pm 0.09$ \\
\hline
\end{tabular}

Table 4: Detection performance for $N=128$ in $\%$ - simulated data.

\begin{tabular}{ccccc}
\hline \multirow{2}{*}{ Number of Features } & \multicolumn{2}{c}{ Naïve-Bayes } & \multicolumn{2}{c}{ MLP } \\
\cline { 2 - 4 } & Class 1 & Class 2 & Class 1 & Class 2 \\
\hline $1-\max _{\mathrm{e}[n]}$ & $87.16 \pm 4.79$ & $98.28 \pm 1.12$ & $87.72 \pm 4.26$ & $98.61 \pm 1.31$ \\
$2-\min _{\mathrm{e}[n],} \max _{\mathrm{e}[n]}$ & $100.00 \pm 0.03$ & $100.00 \pm 0.03$ & $99.95 \pm 0.28$ & $100.00 \pm 0.00$ \\
$3-E_{\mathrm{e}[n]} \min _{\mathrm{e}[n],} \max _{\mathrm{e}[n]}$ & $100.00 \pm 0.00$ & $100.00 \pm 0.00$ & $99.69 \pm 1.04$ & $100.00 \pm 0.00$ \\
$4-\sigma_{\mathrm{e}[n]}^{2}, E_{\mathrm{e}[n]} \min _{\mathrm{e}[n]} \max _{\mathrm{e}[n]}$ & $100.00 \pm 0.00$ & $100.00 \pm 0.00$ & $99.97 \pm 0.14$ & $100.00 \pm 0.00$ \\
$5-\mu_{\mathrm{e}[n]} \sigma_{\mathrm{e}[n]}^{2}, E_{\mathrm{e}[n]} \min _{\mathrm{e}[n]} \max _{\mathrm{e}[n]}$ & $100.00 \pm 0.00$ & $100.00 \pm 0.00$ & $99.98 \pm 0.11$ & $100.00 \pm 0.00$ \\
\hline
\end{tabular}

Table 5: Detection performance for $N=256$ in $\%$ - simulated data.

\begin{tabular}{ccccc}
\hline \multirow{2}{*}{ Number of Features } & \multicolumn{2}{c}{ Naïve-Bayes } & \multicolumn{2}{c}{ MLP } \\
\cline { 2 - 4 } & Class 1 & Class 2 & Class 1 & Class 2 \\
\hline $1-\max _{\mathrm{e}[n]}$ & $98.92 \pm 1.59$ & $99.87 \pm 0.51$ & $98.59 \pm 2.03$ & $99.99 \pm 0.09$ \\
$2-\min _{\mathrm{e}[n]} \max _{\mathrm{e}[n]}$ & $100.00 \pm 0.00$ & $100.00 \pm 0.00$ & $99.62 \pm 1.70$ & $100.00 \pm 0.00$ \\
$3-E_{\mathrm{e}[n]} \min _{\mathrm{e}[n],} \max _{\mathrm{e}[n]}$ & $100.00 \pm 0.00$ & $100.00 \pm 0.00$ & $99.87 \pm 0.42$ & $100.00 \pm 0.00$ \\
$4-\sigma_{\mathrm{e}[n]}^{2}, E_{\mathrm{e}[n]} \min _{\mathrm{e}[n]} \max _{\mathrm{e}[n]}$ & $100.00 \pm 0.00$ & $100.00 \pm 0.00$ & $99.74 \pm 0.64$ & $100.00 \pm 0.00$ \\
$5-\mu_{\mathrm{e}[n]} \sigma_{\mathrm{e}[n]}^{2} E_{\mathrm{e}[n]} \min _{\mathrm{e}[n]} \max _{\mathrm{e}[n]}$ & $100.00 \pm 0.00$ & $100.00 \pm 0.00$ & $99.98 \pm 0.13$ & $100.00 \pm 0.00$ \\
\hline
\end{tabular}


Regarding the number of features used, Figure 4 shows the hit rate tends to increase with the number of features increasing. Overall efficiencies greater than $85.0 \%$ were obtained by both classifiers with only one feature and $N=16$ samples.

\section{For Real Signals}

Table 6 shows the performances of the proposed detectors for the real signals. As the real signals of the database comprise only disturbances, then it is shown the results only for Class 1 . The overall efficiencies are shown in Figure 5.

(a)

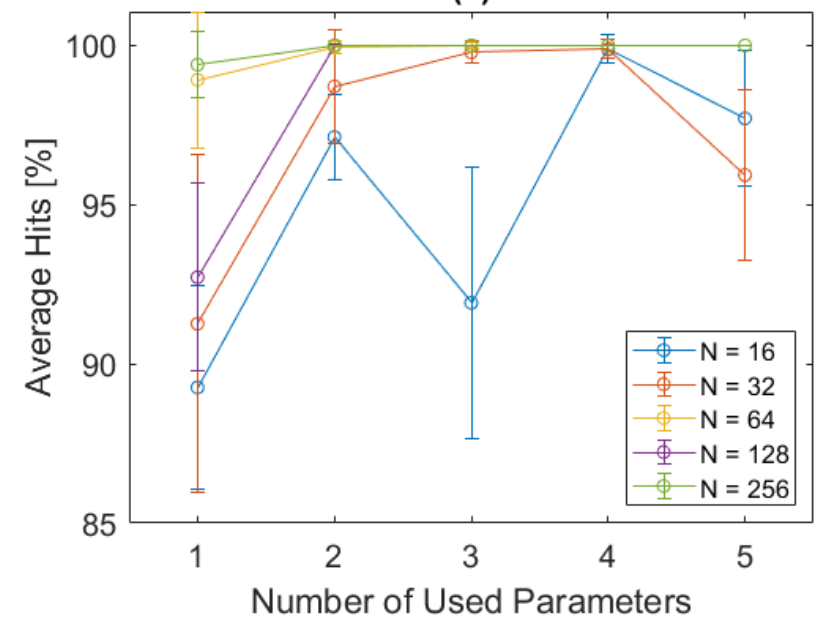

In general, the Naiive-Bayes classifier achieved the best hit rates, showing good generalization capability. With only two features, the NaïveBayes classifier achieved hit rates above $97 \%$ for all window lengths.

\section{Computational Complexity}

The computational complexity of the proposed detection methods can be estimated in terms of the number of mathematical operations required to process a measured signal, which is shown in Table 7 for each step of the proposed method, in terms of the number of samples in the processed signal

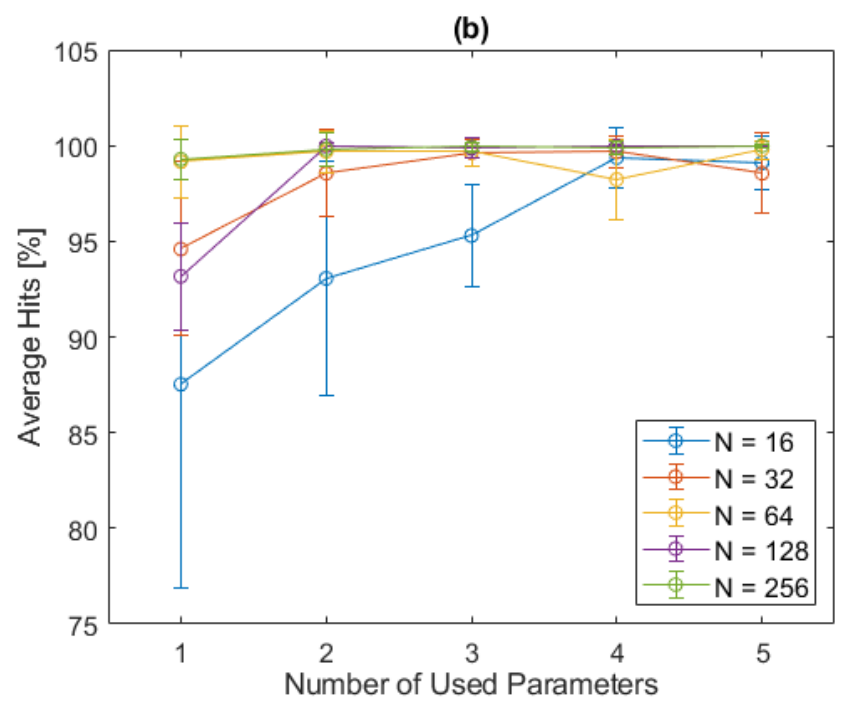

Figure 4: Detection performance (average hits \pm standard deviation) for simulated signals in terms of both the number of features used (from 1 to 5 ) and length of the processed signal window ( $N=16-256)$ for (a) Naïve Bayes classifier and (b) Multi-layer Perceptron.

Table 6: Detection performance for real data.

\begin{tabular}{|c|c|c|c|}
\hline \multirow{2}{*}{ Processing Window } & \multirow{2}{*}{ Number of Features } & Naïve-Bayes & MLP \\
\hline & & Class 1 & Class 1 \\
\hline \multirow{5}{*}{16} & $1-\max _{\mathrm{e}[n]}$ & $81.62 \pm 1.98$ & $68.36 \pm 15.00$ \\
\hline & $2-\sigma_{\mathrm{e}[n]}^{2} \max _{\mathrm{e}[n]}$ & $97.28 \pm 0.09$ & $72.38 \pm 14.57$ \\
\hline & $3-\sigma_{\mathrm{e}[n]}^{2}, \min _{\mathrm{e}[n]}, \max _{\mathrm{e}[n]}$ & $98.14 \pm 0.82$ & $79.53 \pm 5.35$ \\
\hline & $4-\sigma_{\mathrm{e}[n]}^{2}, E_{\mathrm{e}[n]} \min _{\mathrm{e}[n]} \max _{\mathrm{e}[n]}$ & $99.38 \pm 0.43$ & $65.15 \pm 9.30$ \\
\hline & $5-\mu_{\mathrm{e}[n]} \sigma_{\mathrm{e}[n]}^{2} E_{\mathbf{e}[n]} \min _{\mathbf{e}[n]} \max _{\mathbf{e}[n]}$ & $99.65 \pm 0.44$ & $83.38 \pm 7.37$ \\
\hline \multirow{5}{*}{32} & $1-\max _{\mathrm{e}[n]}$ & $83.28 \pm 4.12$ & $74.34 \pm 2.37$ \\
\hline & $2-\min _{\mathrm{e}[n]} \max _{\mathrm{e}[n]}$ & $100.00 \pm 0.00$ & $90.99 \pm 4.69$ \\
\hline & $3-\sigma_{\mathrm{e}[n]}^{2}, \min _{\mathrm{e}[n]} \max _{\mathrm{e}[n]}$ & $99.95 \pm 0.20$ & $91.96 \pm 4.90$ \\
\hline & $4-\sigma_{\mathrm{e}[n]}^{2}, E_{\mathrm{e}[n]}, \min _{\mathrm{e}[n]}, \max _{\mathrm{e}[n]}$ & $100.00 \pm 0.00$ & $84.90 \pm 8.79$ \\
\hline & $5-\mu_{\mathrm{e}[n]} \sigma_{\mathrm{e}[n]}^{2}, E_{\mathrm{e}[n]} \min _{\mathrm{e}[n]} \max _{\mathrm{e}[n]}$ & $100.00 \pm 0.00$ & $86.71 \pm 8.45$ \\
\hline
\end{tabular}


Table 6: Continuation.

\begin{tabular}{|c|c|c|c|}
\hline \multirow{2}{*}{ Processing Window } & \multirow{2}{*}{ Number of Features } & Naïve-Bayes & MLP \\
\hline & & Class 1 & Class 1 \\
\hline \multirow{5}{*}{64} & $1-\max _{\mathrm{e}[n]}$ & $87.28 \pm 0.74$ & $80.08 \pm 2.71$ \\
\hline & $2-\min _{\mathrm{e}[n]}, \max _{\mathbf{e}[n]}$ & $100.00 \pm 0.00$ & $90.30 \pm 12.72$ \\
\hline & $3-E_{\mathrm{e}[n]} \min _{\mathrm{e}[n]} \max _{\mathrm{e}[n]}$ & $100.00 \pm 0.00$ & $95.97 \pm 3.14$ \\
\hline & $4-\sigma_{\mathrm{e}[n]}^{2}, \mathrm{E}_{\mathrm{e}[n]}, \min _{\mathrm{e}[[]]}, \max _{\mathrm{e}[n]}$ & $100.00 \pm 0.00$ & $93.24 \pm 5.54$ \\
\hline & $5-\mu_{\mathrm{e}[n]} \sigma_{\mathrm{e}[n]}^{2} E_{\mathrm{e}[n]}, \min _{\mathrm{e}[n]}, \max x_{\mathrm{e}[n]}$ & $100.00 \pm 0.00$ & $94.45 \pm 4.46$ \\
\hline \multirow{5}{*}{128} & $1-\max _{\mathrm{e}[n]}$ & $89.59 \pm 1.51$ & $87.91 \pm 3.41$ \\
\hline & $2-\min _{\mathrm{e}[n]}, \max _{\mathrm{e}[n]}$ & $99.77 \pm 0.40$ & $81.25 \pm 16.45$ \\
\hline & $3-E_{\mathrm{e}[n]} \min _{\mathrm{e}[n],} \max _{\mathbf{e}[n]}$ & $100.00 \pm 0.00$ & $95.71 \pm 5.02$ \\
\hline & $4-\sigma_{\mathrm{e}[n]}^{2}, E_{\mathrm{e}[n]}, \min _{\mathrm{e}[[]]}, \max _{\mathrm{e}[n]}$ & $100.00 \pm 0.00$ & $94.04 \pm 4.21$ \\
\hline & $5-\mu_{\mathrm{e}[n]} \sigma_{\mathrm{e}[n]}^{2}, E_{\mathrm{e}[n]}, \min _{\mathrm{e}[n]}, \max x_{\mathrm{e}[n]}$ & $100.00 \pm 0.00$ & $86.51 \pm 12.15$ \\
\hline \multirow{5}{*}{256} & $1-\max _{\mathbf{e}[n]}$ & $94.45 \pm 1.72$ & $91.28 \pm 3.65$ \\
\hline & $2-\min _{\mathrm{e}[n]}, \max _{\mathrm{e}[n]}$ & $99.09 \pm 0.00$ & $89.61 \pm 5.89$ \\
\hline & $3-E_{\mathrm{e}[n]} \min _{\mathbf{e}[n]} \max _{\mathbf{e}[n]}$ & $100.00 \pm 0.00$ & $88.78 \pm 8.11$ \\
\hline & $4-\sigma_{\mathrm{e}[n]}^{2}, E_{\mathrm{e}[n]}, \min _{\mathrm{e}[n]}, \max _{\mathrm{e}[n]}$ & $100.00 \pm 0.00$ & $79.24 \pm 12.25$ \\
\hline & $5-\mu_{\mathrm{e}[n]} \sigma_{\mathrm{e}[n]}^{2} E_{\mathrm{e}[n]} \min _{\mathrm{e}[n]} \max _{\mathrm{e}[n]]}$ & $100.00 \pm 0.00$ & $82.57 \pm 14.51$ \\
\hline
\end{tabular}

(a)

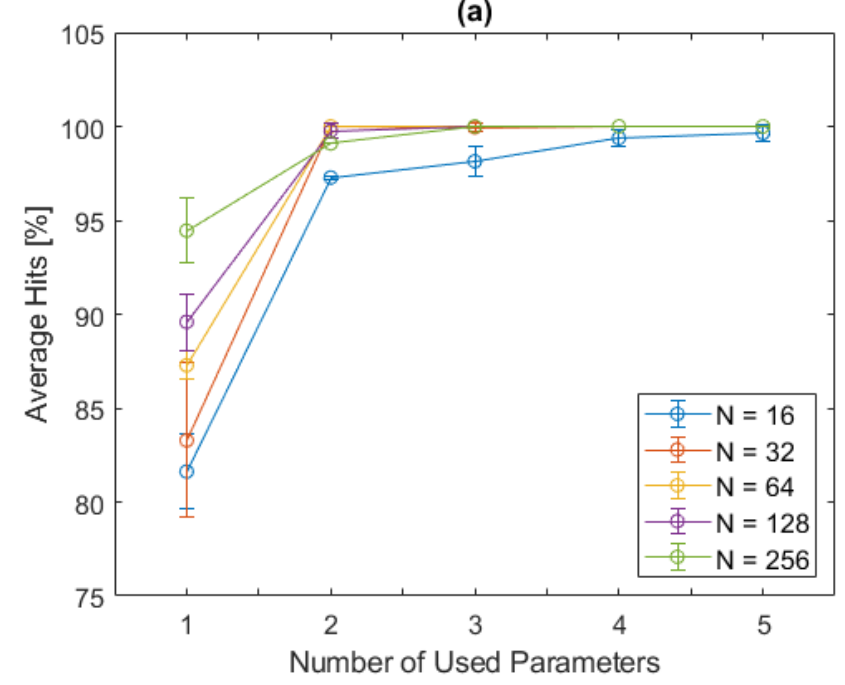

(b)

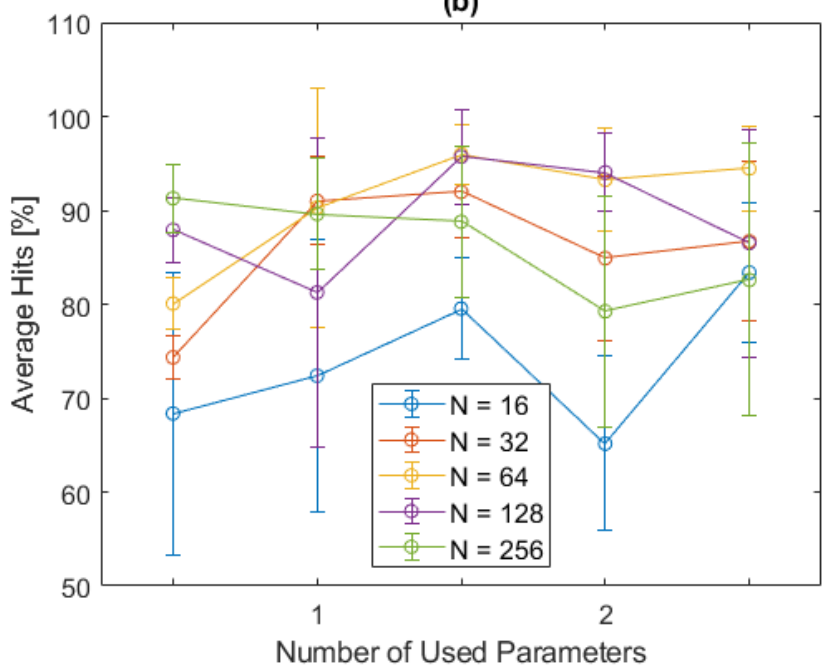

Figure 5: Detection performance (average hits \pm standard deviation) for real signals in terms of both number of features used (from 1 to 5 ) and length of the processed signal window (N=16-256) for (a) Naïve Bayes classifier and (b) Multi-layer Perceptron

Table 7: Number of operations required by the method

\begin{tabular}{ccccc}
\hline \multirow{2}{*}{ Preprocessing } & & Additions & Multiplication & Tanh(.) \\
\hline \multirow{5}{*}{ Extracted features } & Notch Filter & $4 N$ & $3 N$ & - \\
& Normalization & $2 N$ & $N$ & - \\
& $\mu_{\mathrm{e}[n]}$ & $N-1$ & 1 & - \\
& $\sigma_{\mathrm{e}[n]}^{2}$ & $2 N-1$ & $N+2$ & - \\
& $E_{\mathrm{e}[n]}$ & $N-1$ & $N$ & - \\
\hline \multirow{2}{*}{ Classifiers } & $\min _{\mathrm{e}[n]}$ & - & - & - \\
& $\max _{\mathrm{e}[n]}$ & - & $L^{2}+L+3$ & $n_{h}+n_{o}$ \\
\hline
\end{tabular}


window $(N)$, the number of features used by the classifiers $(L)$, and the number of neurons in the hidden layer $\left(n_{h}\right)$ and the output layer $\left(n_{o}\right)$ of the neural network.

The computational complexities of the proposed methods were computed for 16 and 256 samples and for the cases with one and five features for both classifiers (see Table 8). According to Table 8, the NaïveBayes approach presented a computational complexity slightly lower than the MLP. The use of monitored signal segments with $N=$ 16 samples represents a considerable cost reduction in comparison with the use of $N=$ 256 samples.

Table 8: Computational Complexity

\begin{tabular}{cccccc}
\hline \multicolumn{4}{c}{ Naïve-Bayes } & \multicolumn{2}{c}{ MLP } \\
\hline \multirow{2}{*}{$N$} & L & Additions & $\begin{array}{c}\text { Multipli- } \\
\text { cations }\end{array}$ & Additions & $\begin{array}{c}\text { Multipli- } \\
\text { cations }\end{array}$ \\
\hline \multirow{2}{*}{16} & 1 & 100 & 69 & 106 & 70 \\
& 5 & 185 & 115 & 191 & 140 \\
\hline \multirow{2}{*}{256} & 1 & 1540 & 1029 & 1546 & 1030 \\
& 5 & 2345 & 1571 & 2351 & 1580 \\
\hline
\end{tabular}

\section{CONCLUSIONS}

The paper presents a simple disturbance detection method, which is based on a few, easily extracted features, and employ a simple classifier. High detection rates were achieved for both simulated and real signals. Also, the method performs well even when reduced signal windows (up to $1 / 16$ of a cycle of the fundamental component) are considered. The proposed method can be useful in online monitoring systems and for protection purposes due to its low computational complexity. In the context of Smart Grids, where the amount of information and the required $P Q$ levels are very high, the proposed method may contribute as part of an online PQ monitoring system.

\section{ACKNOWLEDGMENTS}

The authors would thank the National Council for Scientific and Technological Development (CNPq-Brazil), the Fundação de Amparo à
Pesquisa do Estado de Minas Gerais (FAPEMIG -Brazil) and the Fundação de Amparo à Pesquisa do Estado do Rio de Janeiro (FAPERJ-Brazil) for supporting this work.

\section{REFERENCES}

BARBOSA, B. H. G.; FERREIRA, D. D. Classification of multiple and single power quality disturbances using a decision tree-based approach. Journal of Control, Automation, and Electrical Systems; 24(5):638-648, 2013. DOI: 10.1007/s40313-0130061-y

CAUJOLLE, M. et al. Reliable power disturbance detection using wavelet decomposition or harmonic model based Kalman filtering. Proceedings of 14th International Conference on Harmonics and Quality of Power - ICHQP 2010, Bergamo, 1-6, 2010. DOI:10.1109/ICHQP.2010.5625309

CHUN-LING, C. et al. Power quality disturbances detection based on Hilbert phase-shifting. Proceedings of Asia-Pacific Power and Energy Engineering Conference - APPEEC 2009, Wuhan, China, 1-4, 2009. DOI:10.1109/ APPEEC.2009.4918613

FERREIRA, D. D. et al. A new power quality deviation index based on principal curves. Electric Power Systems Research, 125:8-14, 2015. DOI:10.1016/j. epsr.2015.03.019

FERREIRA, D. D. et al. Exploiting principal curves for power quality monitoring. Electric Power Systems Research, 100:1-6, 2013. DOI:10.1016/j. epsr.2013.02.006

FERREIRA, D. D.; SEIXAS, J. M.; CERQUEIRA, A. S. A method based on independent component analysis for single and multiple power quality disturbance classification. Electric Power Systems Research, 119:425-431， 2015. DOI:10.1016/j. epsr.2014.10.028

HIRANO, K.; NISHIMURA, S.; MITRA, S. K. Design of digital notch filters. IEEE Transactions on Communications, 22(7):964-970, 1974. DOI:10.1109/ TCOM.1974.1092311

IEEE. Recommended practice for monitoring electric power quality. IEEE Std 1159-2009 (Revision of IEEE Std 1159-1995), 1-81, 2009. 
IEEE. Power Quality Data of the IEEE P1159.3 Task Force, http://grouper.ieee.org/groups/1159/3/ docs.html, this reference was last accessed at 10/12/2012.

LIN, W-M. et al. Detection and classification of multiple power-quality disturbances with wavelet multiclass SVM. IEEE Transactions on Power Delivery, 23(4):2575-2582, 2008. DOI:10.1109/ TPWRD.2008.923463

MARQUES, C. A. G. et al. Improved disturbance detection technique for power-quality analysis. IEEE Transactions on Power Delivery, 26(2):1286-1287, 2011. DOI:10.1109/TPWRD.2010.2092571

MISHRA, S.; BHENDE, C. N.; PANIGRAHI, B. K. Detection and Classification of Power Quality Disturbances Using S-Transform and Probabilistic Neural Network. IEEE Transactions on Power Delivery, 23(1):280-287, 2008. DOI:10.1109/ TPWRD.2007.911125

MITRA, S. K. Digital Signal Processing: A computerbased approach. Third ed., McGraw-Hill, 2005.

MORAVEJ,Z.; ABDOOS, A. A.;PAZOKI, M. Detection and classification of power quality disturbances using wavelet transform and support vector machines. Electric Power Components and Systems; 38(2):182196, 2009. DOI:10.1080/15325000903273387

MORAVEJ, Z.; PAZOKI, M.; ABDOOS, A. A. Wavelet transform and multi-class relevance vector machinesbased recognition and classification of power quality disturbances. European Transactions on Electrical Power; 21(3):212-222, 2011. DOI:10.1002/etep.432

RADIL, T. et al. PQ monitoring system for realtime detection and classification of disturbances in single-phase power system. IEEE Transactions on Instrumentation and Measurement, 57(8):1725-1733, 2008. DOI:10.1109/TIM.2008.925345

RIBEIRO, M. V. et al. Detection of Disturbances in Voltage Signals for Power Quality Analysis Using HOS. EURASIP Journal on Advances in Signal Processing, 2007. DOI:10.1155/2007/59786

RIBEIRO, M. V.; PEREIRA, J. L. R. Classification of single and multiple disturbances in electric signals. EURASIP Journal on Advances in Signal Processing, 2007. DOI:10.1155/2007/56918
RIBEIRO, P. F. et al. Power Systems Signal Processing for Smart Grids, John Wiley and Sons, 2013. DOI: $10.1002 / 9781118639283$

RIEDMILLER, M.; BRAUM, H. A direct adaptive method for faster backpropagation learning: The RPROP algorithm. IEEE International Conference on Neural Networks, 19:586-591, 1993. DOI:10.1109/ ICNN.1993.298623

THAKUR, P.; SINGH, A. K. A novel way to quantify the magnitude of voltage sag. Electrical Engineering, 95:331-340, 2013. DOI: 10.1007/s00202-012-0268-0

THEODORIDIS, S.; KOUTROUMBAS, K. Pattern Recognition. 4 ed., Elsevier, 2009. DOI:10.1016/B9781-59749-272-0.50001-3

YANG, H-T.; LIAO, C-C. A de-noising scheme for enhancing Wavelet-based power quality monitoring system. IEEE Transactions on Power Delivery, 16(3):353-360, 2001. DOI:10.1109/61.924810 